\title{
Trusting the Social Web: issues and challenges
}

\author{
Surya Nepal • Cécile Paris • Athman Bouguettaya
}

Received: 23 July 2013 / Revised: 26 July 2013 /

Accepted: 30 July 2013 / Published online: 10 August 2013

(C) Springer Science+Business Media New York 2013

\section{The Social Web}

The phenomenon of the Social Web (i.e., the Web of Social Media) has caught the attention of research communities in the last decade. Researchers from diverse disciplines ranging from social and behavioral sciences to computer science have started investigating the issues and challenges in the Social Web. Within computer science, researchers from established research areas such as language technologies, machine learning, and service and cloud computing have started looking into the computational and development challenges brought about by the Social Web. With the continued reports of breach of trust and hoax news spreading from social media to mainstream news, trusting the Social Web has become one of the major challenges that need to be addressed. This special issue focuses on this challenge. In this editorial note, we provide a brief introduction of the Social Web, followed by a discussion on the major challenges in building a trusted Social Web. Finally, we present recent work in trusting the Social Web through a summary of the papers included in this special issue.

Social media is increasingly becoming mainstream for a variety of purposes, ranging from online journalism (e.g., blogs), online knowledge bases (e.g., Wikepedia), online marketing (e.g., Twitter), to keeping in touch with friends, family and professional colleagues online (e.g., Facebook, LinkedIn, etc.). These technologies have given rise to a web of social media, also known as the Social Web. The popularity of the Social Web has been overwhelming. It is reported in [8] that $67 \%$ of online adults are connected to one or more social media, and that a large proportion of them first check in to their favorite social media site everyday. This trend is likely to grow as the number of Android-based smartphones shipments alone is expected to reach 1 billion by 2013 [13]. Additionally, the value of social commerce is expected to reach 30 billion dollars within next 5 years. Similarly, $13 \%$ of digital news consumers follow recommendations from Twitter and Facebook. Therefore, the

\footnotetext{
S. Nepal $(\bowtie) \cdot$ C. Paris

CSIRO Computational Informatics (CCI), Marsfield, Australia

e-mail: Surya.Nepal@csiro.au

C. Paris

e-mail: Cecile.Paris@csiro.au
}

\section{A. Bouguettaya}

RMIT University, Melbourne, Australia

e-mail: Athman.Bouguettaya@rmit.edu.au 


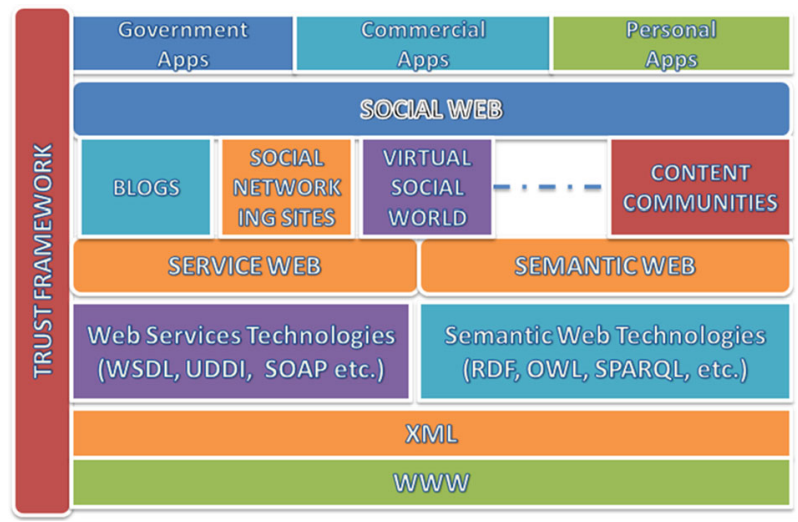

Figure 1 Social Web: technologies, platforms and applications

Social Web is here to stay for the foreseeable future and will revolutionise the way we live, from communicating with our family and friends to conducting businesses. In a nutshell, the Social Web provides a unique platform for digitising our behaviour. This has the enormous potential to change the way we conduct ourselves in the physical world.

There is no consensus about the definition of the Social Web. In what follows, we define the Social Web as the platforms, technologies and applications that enable the Web to support and foster social interactions. The most popular Social Web applications include Twitter, Facebook, LinkedIn and Instagram. Figure 1 shows the key component technologies, platforms and applications that define the Social Web.

Since the introduction of the World Wide Web (WWW or the Web) by Tim Berners-Lee, the Web has gone through a number of evolutions from its initial form - a web of hypertext documents to be viewed by browsers. The early major evolution of the Web was triggered by the introduction of Extensible Markup Language (XML) in 1998 by WWW Consortium (W3C). By the dawn of this century, the Web has established itself as a major place for hosting a variety of applications and data from different domains, ranging from various scientific endeavours to electronic commerce and delivery of government services. W3C has, along with other industry partners, played a significant role in revolutionising the Web on how data and applications are hosted and presented.

The major evolution on developing applications in the Web took place with the introduction of Web Services in 2003 [2]. A Web service is an application that is accessible to other applications over the Web [15]. The vision of Web Services is to develop large scale distributed applications over the Web, where applications can communicate through a standard format similar to how computers can communicate with each other through TCP/IP, using autonomous, transparent software components called services. The foundational technologies for Web Services include Web Services Description Language (WSDL), Universal Description, Discovery and Integration (UDDI) and Simple Object Access Protocol (SOAP). Layers of standards have been proposed as Web Services stack to address other application related issues such as transactions, security and workflows [7]. In recent time, a large number of applications have been developed as services and deployed on the Web. This has changed the Web to a pool of services that can be searched, composed and executed, called the Service Web [1].

In parallel to the evolution of applications on the Web through the Service Web, there has been an evolution of representation of data in the Web through the introduction of the 
Semantic Web [5]. The aim of the Semantic Web is to promote common data formats on the Web with associated semantic content so that the content in the web pages can be linked to each other and processed intelligently. Similar to the three foundational technologies for the Service Web, the Semantic Web is based on the foundational technology: Resource Description Framework (RDF) recommended by W3C; a stack of standards are being developed on top of it [18].

The Service Web and the Semantic Web together provide the fundamental building blocks for the Semantic Web Services. They also enabled the next evolution of the Web: the Web 2.0, with social media as one of its major outcomes [12]. Social media allows the Web to move from data and applications to user interaction and experience. The web of social media that supports and fosters social interactions is called the Social Web.

\section{Building trusted Social Web}

The Social Web provides a unique platform for sharing information and knowledge for individuals, businesses, governments and any organisations. As the Social Web is inherently open and free, it lacks the central control or authority that asserts the trustworthiness of the people and content in it. It is hard to distinguish what is rumor and what is truth. For example, many statesmen/women (e.g., Nelson Mandela, Mikhail Gorbachev, Margaret Thatcher, etc.) and celebrities (e.g., Lady Gaga, Eddie Murphy, etc.) have witnessed their own death in the Social Web. These pieces of fake death news are filtered through into the mainstream newspapers and televisions without much scrutiny and verification due to the high competition of being first to report the news. On the positive side, Social Media has played a major role in providing a universal and unfettered platform for expressing and sharing views to enact political changes (such as the Arab Spring) [10] and providing effective relief in natural disasters (such as the Japan Tsunami and the New Zealand earthquakes) [20], thus consolidating global relief efforts. Furthermore, people are increasingly relying on the Social Web to obtain information on a wide variety of topics, such as travel, healthcare advice and government services [6]. Indeed, the Social Web has become as essential part of the communication eco-system worldwide. Therefore, it is important to address the issue of trust in the Social Web in order to leverage it for the betterment of human society. In the Social Web, there are four essential entities that are involved in trust directly: service consumers, service providers, services and content. In this special issue, we are looking for innovative technologies and solutions from diverse disciplines that address the issue of trust in the Social Web.

Trust has been studied in many disciplines, including Sociology, Psychology, Economics and Computer Science. Each of these disciplines has defined and considered trust from different perspectives, and their definitions may not be directly applicable to the Social Web. In general, trust is a measure of confidence that an entity or entities will behave in an expected manner. In the Social Web, trust has to be studied from different aspects: data (or content), services (or applications), service providers (Web sites, organisations, governments or individuals) and service consumers (organisations or individuals). There has been already work reported in the literature covering these different aspects [11, 19].

Beatty et al. [4] conducted a study of consumer trust on e-commerce Web sites, whereas Grandison et al. [9] surveyed trust from the point of view of applications to identify trust needs for e-commerce applications. Similarly, Artz and Gil [3] provided an overview of trust research in the Semantic Web, whereas Malik and Bouguettaya [14] looked at the issues of 
trust in the Service Web. Josang et al. (2007) published an important survey for Internet applications in which they provided an overview of existing and proposed systems that can be used to derive measures of trust and reputation for Internet transactions.

Review articles focus mainly on trust from a Computer Science perspective. The emergence of the Social Web has spurred new research in the study of trust, and, recently, a number of trust models for social networks have been developed with a specific focus on social aspects of trust. Sherchan et al. presented a comprehensive review of trust in social networks covering literature from both Computer Science and Social Sciences [19].

Trust in the Social Web faces a number of challenges that need to be addressed. We outlined a few fundamental challenges below. First, the issue of trust bootstrapping is paramount, i.e., how to assign a trust value to new entities (e.g., service providers, service consumers, etc.) in the Service Web. Second, the Social Web is built on the social interactions of entities, and hence can be represented as a complex network structure. The propagation of trust and distrust in the networks becomes an important aspect of building trust in the Social Web. Third, recommendation systems are gaining popularity in the Social Web. The recent survey conducted by Forester Research Survey shows that $70 \%$ of US respondents (out of 60,000) trust brands or products recommendations from friends and family. However, trust is low when it comes to consumer written online reviews (46\%). There is thus a scope to develop techniques to increase trust on consumer written online reviews, which in turn helps to build the trusted Social Web. Fourth, the issue of deception must be addressed to build the trusted Social Web, because the Social Web is intrinsically vulnerable to ill-intentioned activities due to anonymity and openness. Deception can occur in many ways including using fake personal identity and wrong content or location. Thus, there is need to develop novel techniques for preventing, detecting, and isolating deceptions in the Social Web. Finally, context plays an important role in building the trusted Social Web. Trust is context sensitive, e.g., a person can trust his/her friends for movie recommendation, but may not trust them for restaurant recommendations, as their taste of food is different. A way to go further in this direction is to build a context-aware trust management framework for the Social Web. We believe that we need to develop a trust framework for the Social Web that can be easily integrated with different technologies, platforms and applications as shown in Figure 1. The W3C initiated a workshop addressing this issue. Passant et al. [16] have proposed a Provenance-Trust-Privacy (PTP) model that deals with three orthogonal, interdependent dimensions: social, interaction and content.

\section{Organisation of the special issue}

This special issue presents six papers that provide the insights into some of the key issues discussed earlier. In the following, we describe the problem space and the contribution summary of each paper.

As stated earlier, the Social Web, through social networks (e.g., Facebook, LinkedIn, etc.), provides a way for people connecting with each other, fostering relationships and offering information and emotional supports. Although people share information and experiences through such sites, privacy disclosure has serious consequences and hence remains a major concern. For example, Stacy Snyder, a student of education at Millersville University, was denied her teaching certificate after campus administrators discovered photos on her MySpace profile portraying her as a "drunken pirate" [17]. There are three major types of privacy disclosure for social networks: content disclosure, identity disclosure and link disclosure. The content disclosure is related to revealing private information about a person 
such as age, sex, gender, and sexual orientation. Identify disclosure is related to identifying a person such as name and social security number. The link disclosure is related to revealing a private link between people in the social networks, such as the link between a person and his/her psychiatrics. The first two types of disclosures have been studied in the context of information systems in general, and the third type of disclosure is very unique to social networks. A standard technique for preserving link privacy is link randomisation. However, such technique causes the structural distortion of the network. The resulting graph cannot be used to analyse the social networks. To overcome this problem, Fard and Wang present a neighbourhood randomisation technique in their paper "Neighbourhood Randomization for Link Privacy in Social Network Analysis". The proposed technique drastically reduces the distortion of the graph structure and yet preserves the privacy of sensitive links.

Informational support in the Social Web can be provided in a variety of ways such as online networks, forums and blogs. In recent times, Q\&A has gained a lot of attention and many people rely on the information available on the Web. However, the accuracy of the information is paramount to maintain the reputation of the Social Web. In many cases, the information provided in the Web may be contradictory, causing ambiguity with respect to its accuracy. This is mainly attributed to the contribution of content from non-experts. In order to address this problem, in this special issue, Pelechrinis et al. propose a solution in their paper "Automatic Evaluation of Information Provider Reliability and Expertise". Their solution is based on the study of human cognitive traits, where each user's activity is monitored by their peers and their compliance to predefined cognitive models is observed. These observations are used to obtain a reliability and expertise consensus for users in the social network.

Allahbakhsh et al. also address the issue of accuracy of information on the Web. In the paper entitled "Robust Evaluation of Products and Reviewers in Social Rating Systems", they looked specifically at the trustworthiness of reviewers and reviews in social rating systems. Social rating systems enable people to rate and provide reviews about various entities (e.g., products, movies, hotels, etc.). These are, in turn, used by web users to make decisions (e.g., what to buy, what movie to watch, what hotel to stay in, etc.). Unfortunately, some abuse these rating systems by posting false or unfair evaluations. In this paper, the authors present a framework and novel algorithms for the robust computation of product rating scores and reviewer trust ranks, even in the presence of unfair reviews. They define a three-pronged approach which includes: a product rating computation that aggregates the community sentiment to assess the quality of a review; an analysis of reviewers' behaviour and a novel method to compute the reviewers' trust rank.

Still addressing the issue of credibility of the information found on the Web, the work of Seth et al. is concerned with participatory media such as blogs. In their paper, "Personalized Credibility Model for Recommending Messages in Social Participatory Media Environments", they propose a method to determine the credibility of messages in order to be able to recommend to users the messages that will be considered the most credible to them. Recommendation systems have been widely used in the Social Web, ranging from recommending news articles to items in e-commerce sites or people for partners and friends. Recommendation systems are often seen as one way to overcome information overload, for example in helping the user in deciding what to read amongst the vast collection of content available. Seth et al.'s work deals with both credibility of information and recommendations. Their proposed approach is inspired by theories from social sciences which show that the people's social context influences their opinions about the credibility of the information they read. Their methodology is based on Bayesian learning and integrates new concepts of context and completeness of messages. 
With recommendation systems playing such an important role on the Social Web, this special issue includes several papers on this topic. Following the paper by Seth et al., the paper by $\mathrm{Wu}$ et al. ("Trust-aware Media Recommendation in Heterogeneous Social Networks") also presents work to recommend to users information sources that are both relevant to the users' interests and come from reliable sources. Wu et al. developed a novel framework for social media recommendation, which they refer to as GCCR, based on the intuition that if a user consistently follows a media provider, one can assume that the user generally trusts that provider. Their approach exploits the historical behaviour of a set of seed users as evidence to assess the trustworthiness of different information providers. The GCCR framework was evaluated with data from Sina Weibo.

Finally, data sparsity and trust are two of the major problems in recommendation systems. One way of addressing the data sparsity problem is to use relevant information collected from social networks for recommendations. The problem of acquiring information from social networks for recommendation is that there is a need to infer trust between people who have not interacted directly with each other in the past. The calculation of trust should also consider the context, as we have already mentioned above. Wang et al. propose a new probabilistic approach, SocialTrust, in their paper "Social Context-Aware Trust Inference for Trust Enhancement in Social Network based Recommendations on Service Providers". The proposed approach considers independent social context (such as personal profile) and dependent social context (such as interactions between two participants).

Acknowledgments The special issue was possible due to the direct and indirect involvements of many researchers, academics, and industry practitioners. We acknowledge and thank the contributing authors. We offer our special appreciation to Springer, to WWW Editors-in-Chief Prof. Professor Yanchun Zhang and Ms. Jennylyn Rosiento from the Springer Journal Editorial Office for helping us to bring this special issue out in a short time frame.

\section{References}

1. Agarwal, S., Handschuh, S., Staab, S.: Surfing the service web. In the semantic web-ISWC 2003, pp. 211-226. Springer Berlin, Heidelberg (2003)

2. Alonso, G., Casati, F., Kuno, H., Machiraju, V.: Web services, pp. 123-149. Springer Berlin, Heidelberg (2004)

3. Artz, D., Gil, Y.: A survey of trust in computer science and the semantic web. Web Semant. 5(2), 58-71 (2007)

4. Beatty, P., Reay, I., Dick, S., Miller, J.: Consumer trust in e-commerce web sites: a metastudy. ACM Comput. Surv. 43(3), 1-46 (2011)

5. Berners-Lee, T., Hendler, J., Lassila, O.: The semantic web. Sci. Am. 284(5), 28-37 (2001)

6. Bista, S.K., Colineau, N., Nepal, S., Paris, C.: The design of an online community for welfare recipients. In Proceedings of the 24th Australian Computer-Human Interaction Conference, pp. 38-41. ACM. (2012)

7. Curbera, F., Khalaf, R., Mukhi, N., Tai, S., Weerawarana, S.: The next step in web services. Commun. ACM 46(10), 29-34 (2003)

8. Duggan, M., Brenner, J.: The demographics of social media users - 2012. PewResearchCenter. (2013)

9. Grandison, T., Sloman, M.: A survey of trust in internet applications. IEEE Commun. Surv. Tutor. 3(4), 2$16(2000)$

10. Howard, P.N., Duffy, A., Freelon, D., Hussain, M., Mari, W., Mazaid, M.: Opening closed regimes: what was the role of social media during the Arab Spring? (2011)

11. Josang, A., Ismail, R., Boyd, C.: A survey of trust and reputation systems for online service provision. Decis. Supp. Syst. 43(2), 618-644 (2007) 
12. Kaplan, A.M., Haenlein, M.: Users of the world, unite! the challenges and opportunities of social media. Bus. Horiz. 53(1), 59-68 (2010)

13. Lam, W.: Cumulative android smartphone shipments will exceed 1 billion in 2013. (2012)

14. Malik, Z., Bouguettaya, A.: Reputation bootstrapping for trust establishment among Web services. IEEE Internet Comput. 13(1), 40-47 (2009)

15. Menasce, D., Almeida, V.: Capacity planning for web services. Prentice Hall, Upper Saddle River, (2001)

16. Passant, A., Kärger, P., Hausenblas, M., Olmedilla, D., Polleres, A., Decker, S.: Enabling trust and privacy on the Social Web. W3C workshop on the future of social networking, Barcelona. (2009)

17. Perez, S.: Social networking profile costs woman college degree. http://readwrite.com/2008/12/04/social network_profile_costs_woman_college_degree\#awesm= obUADLc8Xt2ePJ. Accessed on 18 July $201 \overline{3}$

18. Shadbolt, N., Hall, W., Berners-Lee, T.: The semantic web revisited. IEEE Intell. Syst. 21(3), 96-101 (2006)

19. Sherchan, W., Nepal, S., Paris, C.: A survey of trust in social networks. ACM Comput Surv 45(4). (2013) (forthcoming)

20. Yin, J., Lampert, A., Cameron, M., Robinson, B., Power, R.: Using social media to enhance emergency situation awareness. IEEE Intell. Syst. 27(6), 52-59 (2012) 